\title{
Kinetic Accessibility of Buried DNA Sites in Nucleosomes
}

\author{
Wolfram Möbius, Richard A. Neher, and Ulrich Gerland \\ Arnold Sommerfeld Center for Theoretical Physics (ASC) and Center for Nanoscience (CeNS), \\ LMU München, Theresienstrasse 37, 80333 München, Germany
}

(Received 12 May 2006; published 14 November 2006)

\begin{abstract}
Using a theoretical model for spontaneous partial DNA unwrapping from histones, we study the transient exposure of protein-binding DNA sites within nucleosomes. We focus on the functional dependence of the rates for site exposure and reburial on the site position, which is measurable experimentally and pertinent to gene regulation. We find the dependence to be roughly described by a random walker model. Close inspection reveals a surprising physical effect of flexibility-assisted barrier crossing, which we characterize within a toy model, the "semiflexible Brownian rotor."
\end{abstract}

Although the DNA in eukaryotic cells is packaged into chromatin, its genetic information must be accessible to proteins for read out and processing [1]. The structural organization of chromatin is fairly well known: the fundamental unit is a nucleosome core particle (NCP) consisting of about 150 base pairs (bp) of DNA wrapped in 1.7 turns around a cylindrical histone octamer [2], and NCPs are regularly spaced along the DNA, which is further compactified into higher order structures. In contrast, the conformational dynamics of chromatin is poorly understood. Recent experiments studied these dynamics on the level of individual NCPs using single-molecule force [3] and fluorescence $[4,5]$ techniques. The latter directly observed spontaneous conformational transitions where part of the DNA unwraps reversibly, allowing proteins to access DNA sites that are normally buried. This mode of access, driven by thermal fluctuations, is particularly important for passive DNA-binding proteins, e.g., transcription factors. Here, we study spontaneous DNA unwrapping within a theoretical model; see Fig. 1(a).

Consider a buried DNA site that is accessible only when a DNA segment of length $L$ is unwrapped. How long is the typical dwell time $\tau_{a}$ in the accessible state, i.e., the window of opportunity for protein binding? And what is the typical time $\tau_{i}$ for which it remains inaccessible? $\mathrm{Li}$ et al. [4] measured $\tau_{a}=10-50 \mathrm{~ms}$ and $\tau_{i} \approx 250 \mathrm{~ms}$ for $L \sim 30 \mathrm{bp}$, while Tomschik et al. [5] found $\tau_{a}=$ $100-200 \mathrm{~ms}$ and $\tau_{i}=2-5 \mathrm{~s}$ for $L \sim 60 \mathrm{bp}$. Taken together, these results indicate a significant dependence on $L$ in both time scales, which cannot be reconciled with an early theoretical study [6] suggesting an all-or-none unwrapping mechanism where the nucleosome fluctuates between two conformations only. Instead, these results, as well as previous biochemical experiments [7], imply a multistep opening mechanism.

In this Letter, we propose and characterize a theoretical model for this multistep mechanism, similar in spirit to previous work on histone-DNA interactions which focused mainly on static properties or the calculation of free energy barriers $[6,8,9]$. Within our model, we clarify the physics that determines the $L$ dependence of the time scales $\tau_{a}$ and $\tau_{i}$. We find that the dependence of $\tau_{i}$ can be interpreted with a simple random walker model, which may serve as a fitting model for future experiments that probe the time scales at different $L$ values. In contrast, the $L$ dependence of $\tau_{a}$ reflects the intricate coupling between the DNA polymer dynamics and the dynamics of breaking and reforming DNA-histone contacts. To analyze the effect of this coupling, we introduce a toy model, the "semiflexible Brownian rotor" (SBR); see Fig. 1(b). We identify a generic physical effect of flexibility-assisted barrier crossing, which may arise also in other contexts. It is marked by a characteristic plateau of the time scale at intermediate $L$. Biologically, the $L$ dependence is relevant, because it creates a positioning effect for transcription factor binding sites relative to nucleosomes [10]. We expect that the integration of single NCPs into nucleosome arrays will alter the absolute time scales but not the basic physics of the DNA (un)wrapping process.

Nucleosome model. - The NCP crystal structure [2] shows that both the electrostatic and hydrogen bond interactions between the DNA and the histone complex are
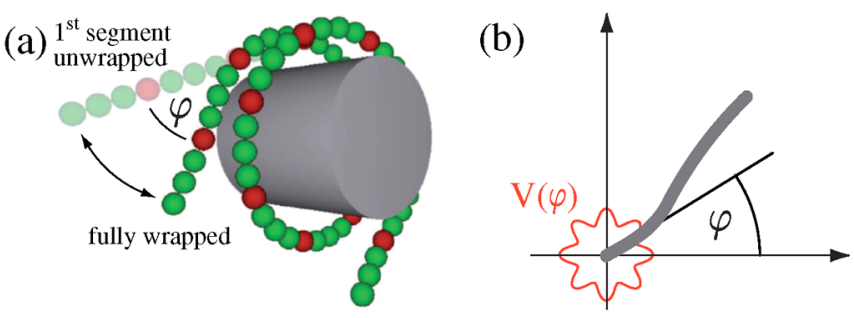

FIG. 1 (color online). (a) Illustration of our nucleosome model. The DNA-histone interaction is localized at contact points attracting the red (dark) beads. The DNA is shown in the ground state as well as a conformation where the first contact is open. (b) Illustration of the semiflexible Brownian rotor (SBR) model. In this toy model, the tradeoff between bending energy and DNA-histone interaction in the nucleosome is mimicked by an angular potential $V(\varphi)$, exerting a torque on the attachment angle $\varphi$ of a semiflexible polymer at the origin. 
mainly localized to 14 contact points, about evenly spaced by $10.2 \mathrm{bp}$ along a superhelical contour with radius $4.2 \mathrm{~nm}$ and helical pitch $2.4 \mathrm{~nm}$. Because we are interested only in the dynamics at a fixed (physiological) salt concentration, we combine the interactions at each of these points into a simple Morse potential [11]. The DNA-histone interaction energy is then

$$
U_{c}=\gamma k_{B} T \sum_{n}\left(1-e^{-\left|\mathbf{r}_{i(n)}-\mathbf{c}_{n}\right| / \rho}\right)^{2},
$$

where $\mathbf{c}_{n}$ is the $n$th contact point on the superhelical contour, $\gamma$ is the depth, and $\rho$ the width of the contact potential. A discrete bead-spring model with beads at positions $\mathbf{r}_{i}$ models the DNA, and $i(n)$ is the bead bound to contact $n$ in the fully wrapped state. The beads are connected by a harmonic potential $U_{s}=\varepsilon_{s} \sum_{i}\left(\mid \mathbf{r}_{i+1}-\right.$ $\left.\mathbf{r}_{i} \mid-a\right)^{2} / 2$ with a typical bead separation $a$ and a stiffness $\varepsilon_{s}$ set to $800 k_{B} T / \mathrm{nm}^{2}$. Below, we use three beads between contacts and at each end (about $2.5 \mathrm{bp} / \mathrm{bead}$ ), unless stated otherwise. Increasing the discretization or $\varepsilon_{s}$ raises the computational effort without affecting our results qualitatively. We account for the bending rigidity of DNA by an energy $U_{b}=\varepsilon_{b} \sum_{i}\left(1-\cos \theta_{i}\right)$ with bending angle $\theta_{i}$ at bead $i$ and a bending stiffness $\varepsilon_{b}$ adjusted such that the apparent persistence length matches the known $\ell_{p} \approx$ $50 \mathrm{~nm}$ for DNA at physiological salt conditions. Furthermore, we incorporate the screened electrostatic self-repulsion of DNA through a Debye-Hückel potential $U_{\mathrm{DH}}=k_{B} T l_{B}(\tau a)^{2} \sum_{i<j} e^{-\kappa\left|\mathbf{r}_{i}-\mathbf{r}_{j}\right|} /\left|\mathbf{r}_{i}-\mathbf{r}_{j}\right|$ with the Bjerrum length $l_{B} \approx 0.7 \mathrm{~nm}$, a charge density $\tau=$ 2 charges/bp, and a screening length $\kappa^{-1} \approx 1 \mathrm{~nm}$. We use a contact radius $\rho=0.5 \mathrm{~nm}$ in between the range of hydrogen bonds and electrostatic interactions and adjust the depth $\gamma$ of the Morse potential to match the binding free energy [12] of $\approx 1.5 k_{B} T$ per contact estimated from biochemical experiments [7,9]. Taken together, the total energy is $U=U_{s}+U_{b}+U_{\mathrm{DH}}+U_{c}$. To study the dynamics of our model, we perform Brownian dynamics simulations with the overdamped Langevin Eqs.

$$
\dot{\mathbf{r}}_{i}(t)=-\mu_{b} \nabla_{\mathbf{r}_{i}} U\left(\left\{\mathbf{r}_{j}\right\}\right)+\boldsymbol{\eta}_{i}(t),
$$

where $\mu_{b}$ is the bead mobility, and the absolute time scale is set by $a^{2} / \mu_{b} k_{B} T$. The random forces $\boldsymbol{\eta}_{i}$ satisfy $\left\langle\boldsymbol{\eta}_{i}(t)\right.$. $\left.\boldsymbol{\eta}_{j}\left(t^{\prime}\right)\right\rangle=6 \mu_{b} k_{B} T \delta_{i, j} \delta\left(t-t^{\prime}\right)$.

Unwrapping dynamics. $-A$ suitable reaction coordinate for the opening of a single contact is the attachment angle $\varphi$, see Fig. 1(a), which changes by $\Delta \varphi \approx 45^{\circ}$ in this process. The equilibrium distribution $p(\varphi)$ for the first contact is shown in Fig. 2(a). Its bimodal form suggests to approximate a contact by a 2-state system, with rates $k_{b}$, $k_{u}$ for binding and unbinding, respectively. To test whether such a reduced description is sufficient, we initiate simulations in the fully wrapped state and determine the functionally relevant time scales, i.e., the average time $\tau_{i}(n)$ until contact $n$ opens to expose the $n$th DNA segment and the average time $\tau_{a}(n)$ until contact $n$ recloses $[13,14]$. The
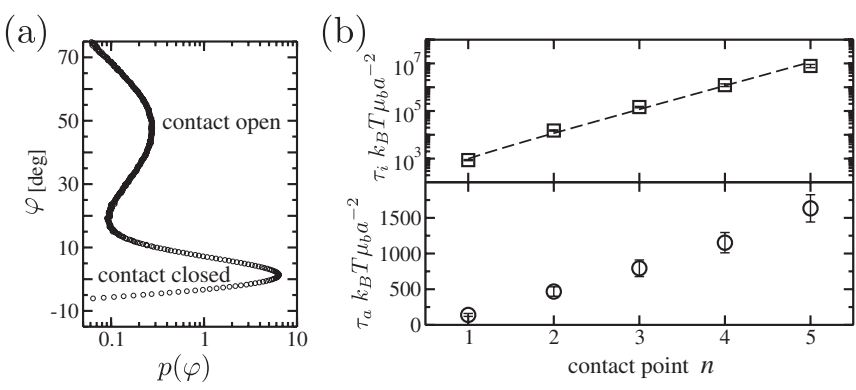

FIG. 2. (a) Equilibrium distribution of the DNA angle $\varphi$ defined in Fig. 1(a). The two peaks at $\varphi=0$ and $\varphi \approx 45 \mathrm{deg}$ correspond to the fully wrapped state and the state with contact 1 open, respectively. (b) Kinetics of DNA site exposure within our nucleosome model. The dwell time in the inaccessible state (squares) increases roughly exponentially with the number of contacts that must open to render a DNA site accessible. The dashed line is a fit to Eq. (3). The circles show the average time the $n$th contact point remains open.

results are shown in Fig. 2(b) for $n \leq 5$ [15]. Within the reduced description of consecutive 2-state contacts, $\tau_{i}(n)$ can be calculated as a mean first passage time [16] for a 1D biased random walker with hopping rates $k_{u}, k_{b}$. The walker starts at site zero (reflecting boundary) and reaches site $n$ after an average time

$$
\tau_{i}(n)=\frac{k_{u}^{-1}}{1-K}\left[\frac{1-K^{n}}{1-K^{-1}}+n\right] \stackrel{K \gg 1}{\approx} \frac{K^{n-1}}{k_{u}} .
$$

Here, $K=k_{b} / k_{u}$ can be interpreted as the effective equilibrium binding constant per contact. The exponential increase of $\tau_{i}(n)$ is clear also from the equivalence of the biased random walk with a random walk against a free energy ramp. The excellent fit of (3) to the simulation data (dashed line) indicates that the reduced description is sufficient for the dwell times in the inaccessible state. In contrast, it proves insufficient for the dwell times in the accessible state, because $\tau_{a}(n)$ in Fig. 2(b) is clearly not constant as one would expect with a fixed binding rate $k_{b}$. Thus, we find $\tau_{a}(n)$ to be a more sensitive probe for the physics of spontaneous site exposure than $\tau_{i}(n)$.

To probe the effect of the DNA length on the rewrapping kinetics, we vary the number of overhanging beads before contact 1 and plot $\tau_{a}(1)$ as a function of the overhang length $L$ in Fig. 3(a). Superimposed is the data of Fig. 2(b) (bottom) with $n$ converted to contour length. The good agreement of these dependencies indicates that $\tau_{a}$ is determined by polymer dynamics. Indeed, we will now see that contact breaking and reformation of a rotating semiflexible polymer displays much richer physics than a simple 1D barrier crossing process.

Semiflexible Brownian rotor.-The essential physics of contact formation in the nucleosome is captured by the toy model depicted in Fig. 1(b): A semiflexible polymer with contour length $L$ and persistence length $\ell_{p}$ is attached to a point about which it can rotate in a plane. The attachment angle $\varphi$ experiences a periodic potential $V(\varphi)=$ $V_{0} \cos (2 \pi \varphi / \Delta \varphi)$, which creates preferred angles sepa- 

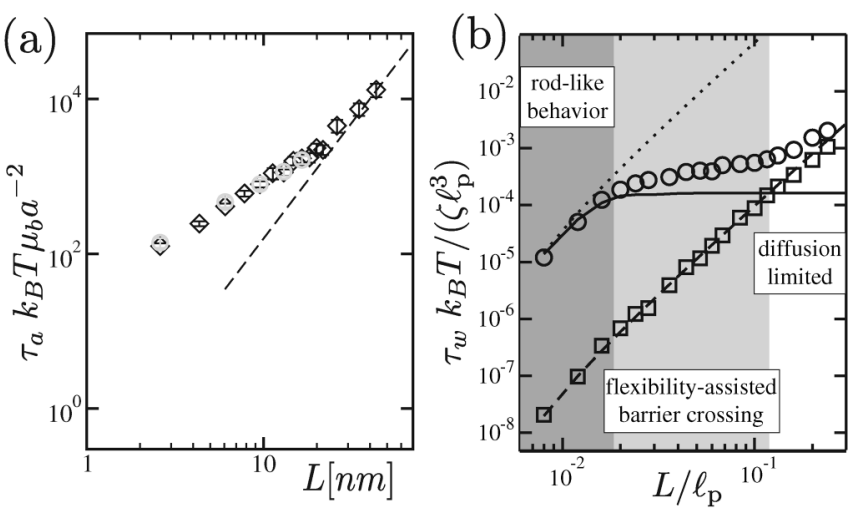

FIG. 3. (a) The dependence of the dwell time $\tau_{a}(n=1)$ on the overhanging DNA length (diamonds) is compatible with $\tau_{a}(n)$ when $n$ is converted to contour length (gray open circles). The dashed line indicates the diffusion limit (see main text for details). (b) The average barrier crossing time $\tau_{w}$ (open circles) for the SBR model of Fig. 1(b). At small lengths, the barrier crossing time follows that of a stiff rod (indicated by the dotted line). Beyond a crossover length $\ell_{c} \ll \ell_{p}$, barrier crossing is much faster than for a stiff rod. For large lengths, $\tau_{w}$ approaches the diffusion limit, i.e., $\tau_{w}$ of the free SBR (open squares). With $L<\ell_{p}$, free diffusion of the SBR is virtually indistinguishable from free diffusion of a rigid rod (dashed line). The crossover from the rodlike regime to the intermediate regime is well described by the theoretical analysis (solid line), see main text.

rated by potential barriers as in our nucleosome model (there, the barrier for contact reformation results from the DNA bending energy and the electrostatic repulsion). The main difference is that the length of the rotating polymer is constant for this SBR, while it changes slightly when a contact breaks or reforms in the nucleosome. Also, we do not consider a directional bias in the SBR, because it is not essential for what follows. So far, barrier crossing of semiflexible polymers was studied only for situations where the entire polymer experiences an external potential [17]. In the NCP, the potential acts only on the angle at the attachment point.

To characterize the phenomenology of the SBR, we determine its barrier crossing rate $1 / \tau_{w}$ with Brownian dynamics simulations of a discrete bead-spring model [18]. The circles in Fig. 3(b) show $\tau_{w}$ as a function of $L / \ell_{p}$ for $V_{0}=5 k_{B} T$. We observe that at very short lengths, $\tau_{w}$ follows the stiff rod behavior $\tau_{w} \sim L^{3}$ [19] indicated by the dotted line. However, above a certain length $\ell_{c}$, there is a regime where $\tau_{w}$ is nearly insensitive to $L$, before it rises again. Hence, for lengths $L>\ell_{c}$ the semiflexible polymer crosses the barrier much faster than the stiff rod. What is the physical mechanism for this acceleration? One effect of a finite flexibility is a reduced mean end-to-end distance (due to the undulations in the contour), which in turn leads to a larger rotational mobility. However, with $V(\varphi)=0$, the rotational diffusion time of a semiflexible polymer over an angle $\Delta \varphi$ (squares) is almost identical to that of a stiff rod (dashed line) when $L<\ell_{p}$. Hence the acceleration is not a mobility effect. Note that the dashed line is also the diffusion limit for $\tau_{w}$, which induces a second crossover from a reaction to a diffusion controlled process. The equivalent diffusion limit is shown also in Fig. 3(a) (dashed line). It indicates that the $\tau_{a}(n)$ data for the nucleosome is indeed in the accelerated barrier crossing regime.

Flexibility-assisted barrier crossing. - To understand the interplay between the polymer dynamics and the barrier crossing dynamics qualitatively, we recall the basic aspects of each: (i) A semiflexible polymer of length $L$ relaxes its conformational degrees of freedom in a time $\sim L^{4} / \ell_{p}$ [20]. Conversely, within a given time $\tau$, a local bending deformation is "felt" only over a length $\ell \sim$ $\left(\ell_{p} \tau\right)^{1 / 4}$. (ii) The probability current over a barrier is proportional to the quasiequilibrium occupancy of the transition state and to the relaxation rate $\tau^{-1}$ out of this state. Together, (i) and (ii) imply that $\ell_{c}$ is the length of the polymer segment that gets deformed during the relaxation process away from the potential peak. We estimate $\ell_{c}$ by noting that the attachment angle relaxes according to $\dot{\varphi}=$ $-\mu\left(\ell_{c}\right) \partial V / \partial \varphi$, where $\mu\left(\ell_{c}\right) \sim \ell_{c}^{-3}$ is the rotational mobility of the deformed segment. Hence, $\tau \sim$ $\ell_{c}^{3}(\Delta \varphi / 2 \pi)^{2} / V_{0}$ and with $\ell_{c} \sim\left(\ell_{p} \tau\right)^{1 / 4}$, we find

$$
\ell_{c}=C \ell_{p} \frac{k_{B} T}{V_{0}}\left(\frac{\Delta \varphi}{2 \pi}\right)^{2}
$$

where $C$ is a constant to be determined below. For lengths below $\ell_{c}$, the entire polymer is involved in the relaxation process, i.e., it behaves like a stiff rod.

Quantitative theory for the crossover.-To render the above picture quantitative, we employ the Langer theory for multidimensional barrier crossing processes [21]. For the case at hand, one can show [22] that the barrier crossing time simplifies to $\tau_{w}=\frac{\pi}{\lambda_{-}} e^{2 V_{0} / k_{B} T}$, where $\lambda_{-}$is the eigenvalue associated with the unstable mode at the saddle point. We calculate $\lambda_{-}$using the continuous wormlike chain model in the weakly bending approximation [23]. At the transition state the chain is straight, e.g., along the $x$ axis. We denote deviations from this configuration by $y(x, t)$. The chain dynamics follows $\partial_{t} y=$ $-\left(k_{B} T \ell_{\mathrm{p}} / \zeta\right) \partial_{x}^{4} y$ with a friction coefficient $\zeta$. With $\Gamma=$ $V_{0}(2 \pi / \Delta \varphi)^{2}$ denoting the curvature of the potential at the transition state, the torque on the attached polymer end is $-\left.\Gamma \partial_{x} y\right|_{x=0}$. This torque must be balanced by a local bend resulting in the boundary condition $\left.k_{B} T \ell_{p} \partial_{x}^{2} y\right|_{x=0}=$ $-\left.\Gamma \partial_{x} y\right|_{x=0}$. The other boundary conditions are $\left.y\right|_{x=0}=$ $\left.\partial_{x}^{2} y\right|_{x=L}=\left.\partial_{x}^{3} y\right|_{x=L}=0$. We find a unique unstable mode with eigenvalue $\lambda_{-}=k_{B} T \ell_{p} \alpha^{4} / 4 \zeta L^{4}$ and $\alpha$ determined by

$$
\frac{\alpha[\sinh (\alpha)-\sin (\alpha)]}{\cosh (\alpha)+\cos (\alpha)+2}=\sqrt[3]{12} \frac{L}{\ell_{c}},
$$

where $\ell_{c}$ is as in (4) with $C=\sqrt[3]{12}$. In the limit $L \ll \ell_{c}$, we find $\lambda_{-}=3 \Gamma / \zeta L^{3}$ independent of the stiffness, whereas in the opposite limit $\lambda_{-}=3 \Gamma / \zeta \ell_{c}^{3}$ independent 

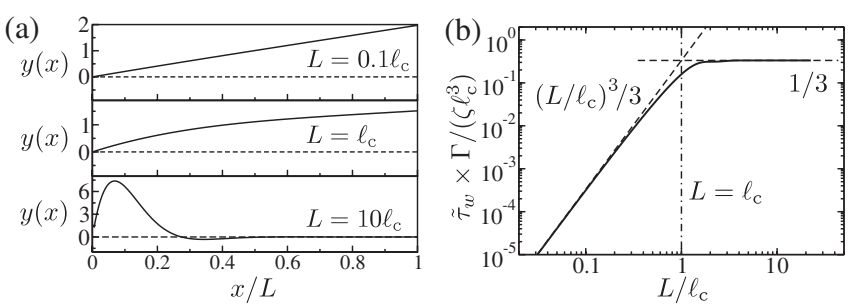

FIG. 4. Dynamics at the barrier. (a) The unstable eigenmode for three different lengths. Polymers shorter than $\ell_{c}$ rotate without significant deformation, while long polymers form a bulge of size $\sim \ell_{c}$ at the origin. (b) The prefactor of the Kramers time $\tilde{\tau}_{w}=1 / \lambda_{-}$as a function of the length. The prefactor increases as $L^{3}$ if $L \ll \ell_{c}$ and is constant if $L \gg \ell_{c}$.

of $L$. Figure 4 shows (a) the unstable eigenmode for $L / \ell_{c}=\{0.1,1,10\}$ and (b) the crossover in the barrier crossing time. The eigenmode shape confirms our qualitative picture: stiff and short polymers respond to the torque by rotating as a whole, whereas the torque shapes a bulge of size $\sim \ell_{c}$ in longer polymers. For a discrete polymer model, the same analysis can be performed, but the eigenvalue $\lambda_{-}$must be computed numerically. The solid line in Fig. 3 shows the resulting barrier crossing time for the same discretization as used in the Brownian dynamics simulations of the SBR model. Indeed, the crossover from the rodlike to the flexibility-assisted barrier crossing is well described by this analysis. The deviations at larger $L$ can be attributed to finite barrier corrections [24].

Discussion and outlook. - The experiments [4,5] have shown that the functionally relevant time scales $\tau_{i}$ and $\tau_{a}$ depend on the position on the nucleosomal DNA. Our theoretical study suggests that these time scales additionally depend on the total DNA length. The position dependence of $\tau_{i}$ should follow the random walker model (3), which is the minimal model for a gradual, multistep opening mechanism. However, we expect that the positiondependence of $\tau_{a}$ and the length-dependence of both time scales will reflect the polymer dynamics of the DNA. Within our toy model, the semiflexible Brownian rotor, we find three physically distinct regimes for this length dependence; see Fig. 3(b). The intermediate regime displays a striking flexibility-assisted barrier crossing effect, the onset of which is marked by the new length scale $\ell_{c}$ of Eq. (4). It can be interpreted as the length over which the polymer contour is deformed as it passes over the potential barrier. Because $\ell_{c}$ is considerably smaller than the persistence length $\ell_{p}$, we expect that the onset of the intermediate regime will not be detectable in nucleosomes. However, nucleosomes should display the crossover from flexibility-assisted barrier crossing to diffusion-limited dynamics as shown in Fig. 3(a). All three regimes of Fig. 3(b) could be probed in an experimental realization of the SBR model, e.g., with an actin filament as the rotating polymer.

We thank H. Boroudjerdi, T. Franosch, E. Frey, O. Hallatschek, S. Leuba, R. Netz, R. Phillips, P. Reimann, P. R. ten Wolde, and J. Widom for useful discussions, and the $D F G$ for financial support.

[1] K. Luger and J. C. Hansen, Curr. Opin. Struct. Biol. 15, 188 (2005).

[2] K. Luger et al., Nature (London) 389, 251 (1997).

[3] B. D. Brower-Toland et al., Proc. Natl. Acad. Sci. U.S.A. 99, 1960 (2002).

[4] G. Li, M. Levitus, C. Bustamante, and J. Widom, Nat. Struct. Biol. 12, 46 (2005).

[5] M. Tomschik, H. Zheng, K. van Holde, J. Zlatanova, and S. Leuba, Proc. Natl. Acad. Sci. U.S.A. 102, 3278 (2005).

[6] N.L. Marky and G. S. Manning, J. Mol. Biol. 254, 50 (1995).

[7] K. Polach and J. Widom, J. Mol. Biol. 254, 130 (1995).

[8] K. Kunze and R. Netz, Phys. Rev. Lett. 85, 4389 (2000); I. Kulic and H. Schiessel, ibid. 91, 148103 (2003); 92, 228101 (2004); W. Li, S.-X. Dou, and P.-Y. Wang, J. Theor. Biol. 230, 375 (2004); D. Beard and T. Schlick, Structure 9, 105 (2001).

[9] H. Schiessel, J. Phys. Condens. Matter 15, R699 (2003).

[10] E. Segal et al., Nature (London) 442, 772 (2006).

[11] Nucleosomal DNA is slightly unwound [2], with a small torsional energy $\sim 1 k_{B} T$ per $10 \mathrm{bp}$. We include this twist contribution into the effective contact potential.

[12] Note that our free energy cost per contact is slightly larger for the first contact and considerably larger when less than one turn of the superhelix remains wrapped, due to the electrostatic self-repulsion of DNA.

[13] Because spontaneous unwrapping for $n>2$ is rare, we use the forward flux sampling method to reduce the computational effort; see R. J. Allen, D. Frenkel, and P. R. ten Wolde, J. Chem. Phys. 124, 024102 (2006).

[14] We exclude transient wrapping or unwrapping events, i.e., we only count transitions that reach the metastable state in the neighboring potential valley.

[15] For $n>5$ the electrostatic self-repulsion of the DNA is less significant. This stabilizes the remaining contacts and modifies the exposure kinetics of the central sites (close to the dyad) [22].

[16] C. Gardiner, Handbook of Stochastic Methods (Springer, Berlin, 1983).

[17] P. Kraikivski, R. Lipowsky, and J. Kierfeld, Europhys. Lett. 66, 763 (2004).

[18] The time $\tau_{w}$ is operationally defined through the long-time rotational diffusion coefficient $D=\Delta \varphi^{2} / 2 \tau_{w}$ over many potential wells. Thus, $\tau_{w}$ measures the time for barrier crossing events that involve not only the passage of $\varphi$ to a neighboring valley in $V(\varphi)$, but also turning of the polymer tip into the direction of the new valley, analogous to $\tau_{a}$ in the nucleosome model [14].

[19] M. Doi and S. Edwards, The Theory of Polymer Dynamics (Clarendon Press, Oxford, 1986).

[20] L. Le Goff, O. Hallatschek, E. Frey, and F. Amblard, Phys. Rev. Lett. 89, 258101 (2002).

[21] J. Langer, Phys. Rev. Lett. 21, 973 (1968).

[22] W. Möbius, R. A. Neher, and U. Gerland (to be published).

[23] See, e.g., C. H. Wiggins, D. Riveline, A. Ott, and R.E. Goldstein, Biophys. J. 74, 1043 (1998).

[24] P. Talkner, Chem. Phys. 180, 199 (1994). 